\title{
Fontes de valores morais na cultura do Design Colaborativo
}

\author{
Sources of moral values in Collaborative Design culture
}

DA SILVA, Tânia Luisa Koltermann; Doutorada; Universidade Federal do Rio Grande do Sul

tania.koltermann@ufrgs.br

PAROLIN, Guilherme; Graduado; Universidade Federal do Rio Grande do Sul

guilherme.parolin@outlook.com

VAN DER LINDEN, Julio Carlos de Souza; Pós-doutorado; Universidade Federal do Rio Grande do Sul

julio.linden@ufrgs.br

\section{Resumo}

Valores morais refletem-se em crenças nutridas, práticas repetidas e artefatos utilizados pelos indivíduos. Na medida em que a disciplina do design tem como cerne formatar esses mesmos artefatos, também possui um papel central no fomento e ressignificação dos valores cultivados pelos usuários. A partir de uma pesquisa bibliográfica aliando valores morais à disciplina do design, esta pesquisa busca organizar e ampliar o conhecimento sobre as fontes de valores morais no processo de design colaborativo, em que usuários e outros stakeholders tem participação ativa no processo de projeto. Inicialmente, são explorados os valores mais relevantes à prática do design, sua categorização e seus papéis no processo criativo. Por fim, são identificadas e exploradas em maior detalhe quatro grandes categorias de fontes de valores morais no processo de design colaborativo: o artefato; o designer; o usuário e outros stakeholders; e o processo.

Palavras Chave: valores morais; design colaborativo; cultura profissional.

\begin{abstract}
Moral values are reflected in beliefs fostered, practices repeated and artifacts used by individuals. As far as the design discipline has as its core the formatting of those same artefacts, it also has a central role in promoting and resignifying moral values fostered by users. From a bibliographic research that allied moral values to the design discipline, this research sought to sort and widen the knowledge about the sources of moral values in a collaborative design process, in which users and other stakeholders have an active participation in the project. Initially, it explores the most relevant values to the design practice, its categorization and roles in the creative process. Lastly, it identifies and explores in greater detail four big categories of sources of moral values in the collaborative design process, which are: the artifact; the designer; the user and other stakeholders; and the process.
\end{abstract}

Keywords: moral values; collaborative design; professional culture. 


\section{Introdução}

O significado original e mais usual de valor origina-se na economia clássica (KLAMER, A. 2003). Ainda assim, diversos estudos desse próprio campo já buscavam trazer à tona a importância significados morais de valor, como o do valor confiança no funcionamento das economias de mercado (FUKUYAMA 1996 apud SIEKMANN 1998) e da construção do entendimento de valores como "recursos morais" que geram retorno financeiro (FRANK 1988 apud KLAMER 2003). Em um sentido mais amplo e multidisciplinar, a ideia de valor adquire um significado particular e subjetivo, referindo-se, de modo geral, ao que as pessoas, individual ou coletivamente, consideram bom ou importante em suas vidas (CUMMINGS 2006)(STEEN; VAN DE POEL 2012)(FRIEDMAN; KAHN; BORNING 2006). Valores podem ser assim entendidos, de maneira mais abrangente, como crenças duradouras que representam ideias e/ou qualidades que definem quais modos de conduta ou estados de existência são bons, preferíveis, apoiáveis ou, ainda, oponíveis. Assim, balizam o comportamento, julgamentos, atitudes e comparações frente a objetos e situações específicas (IVERSEN; HALSKOV; LEONG 2012)(HALLORAN et. al 2009).

No que tange à sua aplicabilidade prática no contexto do design, valores possuem uma miríade de usos na disciplina do design, podendo servir como inspiração ao projeto (FLANAGAN; HOWE; NISSENBAUM 2008); fontes de restrições que fornecem direcionamentos à inovação (KNOBEL; BOWKER 2011); objetivos fundamentais; fontes de inovação; fontes de informação ao processo; ou ainda filtros para delimitação de critérios de avaliação de protótipos (COCKTON 2004)(HALLORAN et. al 2009)(SHILTON 2013).

A relevância dos valores ao campo do design se dá na medida em que valores emergem de redes complexas de custos e benefícios (COCKTON 2008). Dado que o processo de design é permeado por uma rede complexa de stakeholders e requisitos de projeto comumente contraditórios, é natural que valores estejam presentes (DORST 2015). Sua importância se comprova na medida em que, dentre os ingredientes para um design bem sucedido, como inovação, experimento psicológico e entendimento contextual, está justamente a entrega de um valor proposital (COCKTON 2004). A incorporação de valores ao design se justifica na medida em que informa e guia o projeto de forma mais completa, trazendo a inteligência ética junto das usualmente já presentes inteligências de negócios e técnica (MANDERS-HUITS; ZIMMER 2009).

A partir deste entendimento geral inicial sobre o conceito de "valores" e como o mesmo se relaciona ao campo do design, esta pesquisa busca, a partir de uma pesquisa bibliográfica com foco na aplicação de valores à disciplina do design, organizar e ampliar o entendimento quanto às fontes de valores morais no processo de design colaborativo, em que usuários ou outros stakeholders tem participação ativa. Isso significa compreender o que são valores e como eles se manifestam especificamente nesse contexto (o "o que"), quais os benefícios que trazem ao processo (o "por quê"), quais os métodos e técnicas utilizados para sua incorporação nos artefatos por meio do processo de design (o "como"), e, finalmente, de onde surgem (o "quem", "onde" e "quando"). Sendo assim, a primeira seção aborda uma conceituação geral mais detalhada sobre valores no processo de design, explorando as características específicas assumidas pelos mesmos nesse contexto, assim como suas potenciais e principais aplicações. A seção seguinte, por sua vez, desenvolve em detalhe as fontes de valores morais no processo de design.

Ressalta-se que, ao longo do presente artigo, é adotado o termo artefato como referindo-se ao resultado projetual de uma atividade de design, seja ele um sistema, produto, serviço ou experiência. Igualmente, o termo valor é utilizado como referindo-se especificamente a 
valores morais, distinguindo-se assim das conotações mais usuais para o termo valor, como elemento que denota valor financeiro (conforme usualmente definido pelo campo da economia clássica) ou no sentido da experiência de consumo (conforme usualmente definido pelo campo do marketing).

\section{Valores relevantes ao Design Colaborativo}

Valores compreendem uma amplitude de conceitos, sendo ao mesmo tempo flexíveis e altamente sujeitos à interpretações individuais. Todavia, valores também estão interconectados e vêm em aglomerados (KLAMER 2003). A categorização de valores mais usual é a que os divide entre valores intrínsecos e extrínsecos. Valores "intrínsecos", ou "finais", seriam valores que são perseguidos por si só (como crescimento pessoal, servir à comunidade e ter relações satisfatórias com outros), enquanto valores "extrínsecos", ou "instrumentais", seriam aqueles que têm importância por ajudarem a atingir outros valores (como alcançar sucesso monetário, ter status e fama e ser atraente) (STEEN; VAN DE POEL 2012)(VAN DE POEL 2014).

Outras classificações de valores dividem-los ainda segundo sua: (1) natureza, sendo "explícitos" (conscientes, quando só emergem quando os usuários são desafiados por outros usuários) ou "implícitos" (inconscientes, quando emergem pelos desenvolvimentos do processo de design) (HALLORAN et. al 2009); (2) expressabilidade, sendo "descritivos" (aqueles que stakeholders consideram importantes) ou "normativos" (aqueles que deveriam ser considerados importantes) (MANDERS-HUITS 2011); (3) abrangência ou interesse, segundo a qual podem ser considerados "públicos" (a população em geral) ou "privados" (grupos de usuários ou mesmo indivíduos específicos)(HALLORAN et. al 2009); ou (4) reducionismo, segundo a qual valores podem ser "monistas" (quando há somente um valor do qual todos os outros advém) ou "pluralistas" (segundo a qual valores não podem ser reduzidos para outros)(VAN DE POEL 2014). Outros autores ainda estabelecem categorias mais variadas para valores, como as expostas no Quadro 1.

Quadro 1 - Categorizações de valores.

\begin{tabular}{cccc}
\hline COCKTON (2004) & $\begin{array}{c}\text { THROSBY (2001) apud } \\
\text { KLAMER (2003) }\end{array}$ & KLAMER (2003) & $\begin{array}{c}\text { STEEN; VAN DE POEL } \\
\text { (2012) }\end{array}$ \\
\hline $\begin{array}{c}\text { Políticos, pessoais, } \\
\text { organizacionais, culturais, } \\
\text { experenciais, espirituais }\end{array}$ & $\begin{array}{c}\text { Estéticos, espirituais, } \\
\text { sociais, simbólicos, } \\
\text { históricos }\end{array}$ & $\begin{array}{c}\text { Econômicos, culturais, } \\
\text { sociais, morais }\end{array}$ & $\begin{array}{c}\text { Instrumentais, } \\
\text { econômicos, morais, } \\
\text { culturais, estéticos }\end{array}$ \\
\hline
\end{tabular}

Fonte: adaptado de vários autores

Já no que tange à valores individuais propriamente ditos, a teoria clássica delimita 10 valores distintos: benevolência, conformidade, tradição, segurança, poder, realização, hedonismo, estimulação, autonomia e universalismo (KLAMER 2003). No entanto, a relevância relativa de cada um varia enormemente entre disciplinas e, especialmente com relação ao design, alguns autores já delimitaram valores que podem ser mais relevantes. Dentre eles, Oreg e Nov (2008) determinaram os valores de autonomia, poder e universalismo como especialmente relevantes para a colaboração em projeto de design de software de código aberto, enquanto que Van de Poel (2014) cita valores como efetividade, eficiência, confiabilidade, robustez, manutenibilidade, compatibilidade e qualidade como os valores instrumentais de maior aplicação ao design, 
enquanto segurança, saúde, bem-estar humano, sustentabilidade, democracia e inclusão consistiram nos valores finais mais relevantes.

De maneira geral, valores são flexíveis e amplamente dependentes de seu contexto (COCKTON 2004). Portanto, e em conformidade ao caráter amplamente situado do design colaborativo (DITTRICH; ERIKSÉN; WESSELS 2014), não é plausível delimitar com precisão um conjunto universal de valores que seja aplicável a todas as situações. Ainda assim, a tomada de conhecimento de um panorama geral das categorias e valores usualmente aplicados à disciplina do design facilita o estabelecimento de um guia geral que embase a escolha dos mais relevantes na medida do contexto específico de cada projeto. A partir do entendimento aqui firmado quanto às categorias e valores mais relevantes, a seção seguinte aborda as fontes de valores no Design Colaborativo.

\section{Fontes de valores no Design Colaborativo}

A partir da pesquisa bibliográfica realizada, é possível destacar quatro grandes grupos de fontes de valores no processo de design. A seguir, cada um deles é explorado em maiores detalhes.

\subsection{Valores vêm dos artefatos}

Uma primeira categoria de fontes de valores no design trata do próprio produto, experiência ou sistema projetado, ou, no termo aqui adotado, o "artefato". Diversos autores frisam os artefatos como sendo maiores do que a soma de suas partes, especialmente por agregarem valores e características simbólicas por meio de sua apreciação e uso (FRIEDMAN; KAHN 2002)(FRIEDMAN; KAHN; BORNING 2006).

Fatores como as experiências dos usuários e os resultados de uso são entendidos como atributos constituintes de artefatos no mesmo grau de importância de características mais objetivas, como materiais, recursos e qualidades físicas do mesmo (COCKTON 2008). A relação entre artefatos e valores é também tão próxima quanto mutuamente dependente: ao mesmo tempo em que valores originam-se dos artefatos, estes dão forma àqueles (HALLORAN et. al 2009)(SENGERS et al. 2005)(FLANAGAN; HOWE; NISSENBAUM 2005).

Sob um olhar do campo da cultura, artefatos podem ser entendidos como um meio de transmissão inconsciente de valores sociais às pessoas (HALLORAN et. al 2009) e de fortalecimento desses mesmos na cultura em que se inserem. Valores corresponderiam a um nível intermediário, de mediação, entre os níveis mais externo ("artefatos") e mais interno ("premissas básicas") da cultura (SPENCER-OATEY 2012). Sob essa visão, artefatos são manifestações ou consequências visíveis dos valores de dada cultura (SPENCER-OATEY 2012). Consistindo a Cultura de uma esfera de valores compartilhados e que conferem distinção de outras culturas, os valores possuem um papel central no processo de construção de sentido e significado para o ser humano (KLAMER 2003).

No contexto específico do design colaborativo, valores podem ser transmitidos aos usuários ou outros stakeholders em meio às atividades colaborativas de projeto pelos artefatos (KNOBEL; BOWKER 2011). Os designers, além de trazerem seus próprios valores ao processo, como é abordado na seção seguinte, possuem um papel central na formatação de tais artefatos intermediários, ou boundary objects, que servem como elementos de ligação entre disciplinas distintas e, assim, permitem a troca mútua de conhecimentos e valores durante o projeto (BROBERG; ANDERSEN; SEIM 2011). Artefatos intermediários podem ser projetados especialmente 
de modo a proporcionar uma "atitude de especulação" pelos usuários, provocando os mesmos a explorarem e (re)avaliarem seus próprios valores (HALLORAN et. al 2009).

\subsection{Valores vêm dos designers}

Adicionalmente aos artefatos, valores também originam-se pelos próprios designers. Em um nível individual, os valores trazidos pelos designers surgem por meio de seu julgamento antes, durante e depois do projeto, o qual advém em grande parte de seu repertório individual de ideias, expectativas e valores próprios (IVERSEN; HALSKOV; LEONG 2012). Em um nível mais geral, no entanto, os valores dos designers são realizados também a partir de seus próprios códigos profissionais, os quais expressam os valores-chave da profissão: integridade e competência na condução dos projetos, respeito às obrigações com clientes e empregadores e responsabilidade para com o público e a sociedade (VAN DE POEL 2014). Os valores trazidos à tona pelos designers podem ser observados especial e explicitamente pela análise das interpretações de descrições de aplicações da solução em desenvolvimento em materiais de projeto, como sketches (STEEN; VAN DE POEL 2012).

No projeto de Design Colaborativo, os variados papéis assumidos pelo designer também são fonte de valores. Dentre esses, o papel de "defensor de valores" é um dos mais proeminentes. O mesmo contempla uma preocupação pessoal com valores, advinda de conhecimento teórico e prático a respeito do tema, que é repassada e reforçada à equipe de projeto, podendo variar de um caráter autoritário a um de acompanhamento passivo para conscientização e aconselhamento (MANDERS-HUITS; ZIMMER 2009). Outro papel de relevância, de caráter mais abrangente, é a atuação do designer como "praticante reflexivo", a qual compreende participar em um diálogo com as próprias ações por meio do entendimento do problema de design e da tomada de consciência de discrepâncias entre as próprias crenças (ou valores) e ações (FLANAGAN; HOWE; NISSENBAUM 2008).

É válido destacar, no entanto, que valores pessoais advindos das crenças dos designers comumente conflitam com os objetivos de projeto (FLANAGAN; HOWE; NISSENBAUM 2005). Na medida em que o mercado apresenta um foco em valores instrumentais como máxima eficiência, produtividade e lucratividade, o designer deve confrontar-se com a questão da supremacia de seus valores morais pessoas sobre esses, engajando um processo reflexivo através do qual possa lidar com trade-offs inerentes à essa tarefa (MANDERS-HUITS; ZIMMER 2009)(MANDERS-HUITS 2011). Além disso, no design colaborativo, seus valores também podem conflitar especialmente com os valores priorizados ou trazidos pelos usuários e outros stakeholders, os quais são abordados como fontes de valores na seção seguinte.

\subsection{Valores vêm dos usuários e outros stakeholders}

Ainda que os designers projetam recursos ou propriedades que propositalmente apóiam certos valores em detrimento de outros, o uso do artefato depende também essencialmente dos objetivos dos usuários (FRIEDMAN; KAHN; BORNING 2006). O contexto em que os objetos se encontram são tão determinantes para sua percepção (tanto física quanto mesmo simbólica ou de significado) quanto das suas características físicas reais. Dessa forma, é possível afirmar que praticar design vai além de conceituar e realizar artefatos, também criando as relações das coisas com os indivíduos.

Em um processo de design colaborativo, valores não são definidos por si só, mas descobertos especialmente a partir da comunicação com pessoas (COCKTON 2004), em um 
processo de imersão dinâmico e de diálogo entre designers e stakeholders (IVERSEN; HALSKOV; LEONG 2012) em que estes últimos são desafiados (HALLORAN et al. 2009) (IVERSEN; HALSKOV; LEONG 2012). Os stakeholders, envolvendo Indivíduos, instituições, sociedades e culturas, sugerem valores ou levantam demandas orientadas a valores de importância para si (COCKTON 2008) (FLANAGAN; HOWE; NISSENBAUM 2008). Nesse contexto, valores são sujeitos a diferentes interpretações (KLAMER 2003), possuindo uma linguagem própria e conceitualizações dentro de seu campo respectivo (FRIEDMAN; KAHN; BORNING 2006), e assumem matizes específicos para cada indivíduo, disciplina ou local (HALLORAN et. al 2009). O papel do designer nesse cenário é o de integrar todos os atores sociais de relevância na análise de futuros artefatos por meio de um debate democraticamente organizado (VERBEEK 2006).

Os valores aspirados pelos usuários são especialmente difíceis de serem observados diretamente. Usuários expressam valores implicitamente, de maneiras menos abstratas do que os encontrados nas taxonomias, mas mais concretos e práticos, baseados em situações e conflitos reais (HALLORAN et. al 2009). Dessa forma, assim como os designers, aquilo que os usuários trazem para o processo, tais como artefatos, ideais, inspirações ou trabalhos prévios, influencia o tipo de valor que é priorizado (IVERSEN; HALSKOV; LEONG 2012). Os valores dos usuários e outros stakeholders podem ser explorados através de, por exemplo, interpretações de declarações em entrevistas com stakeholders chave ou grupos focais (STEEN; VAN DE POEL 2012)(SPENCER-OATEY 2012); análise de conteúdo de documentos e outros artefatos (SPENCER-OATEY 2012); testes colaborativos de protótipos (FLANAGAN; HOWE; NISSENBAUM 2008)(SHILTON 2013); ou mesmo o próprio trabalho em equipes interdisciplinares (SHILTON 2013). Dentre esses métodos, é dado especial destaque à elaboração e teste colaborativos de protótipos como possuindo um papel essencial para descobrir os valores dos usuários e outros stakeholders (FLANAGAN; HOWE; NISSENBAUM 2008). O teste de e com protótipos pode inclusive apontar consequências ou valores inesperados (VAN DE POEL 2014).

As diferenças entre os valores pretendidos pelos designers e aspirados pelos usuários podem ser uma grande fonte de conflito no processo de design (STEEN; VAN DE POEL 2012). As mesmas podem vir tanto de partida quanto desenvolverem-se ao longo do projeto, visto que uma das características chave dos valores é que eles diferem tanto com o tempo, na medida em que os designers, usuários e outros stakeholders reavaliam suas importâncias (FLANAGAN; HOWE; NISSENBAUM 2005) quanto de indivíduo para indivíduo, os quais costumam manter-se aferrados a seus valores fortemente (KNOBEL; BOWKER 2011). Conflitos entre valores podem surgir, assim, de trade-offs entre valores diferentes que não possam ser trabalhados simultaneamente (MANDERS-HUITS 2011)(STEEN; VAN DE POEL 2012); de conflitos entre objetivos de projeto e das motivações dos designers (DORST 2015); de conflitos na posição de papel do designer como autor criativo ou a serviço de um cliente (DORST 2015); de conflitos com valores trazidos pelos requisitos ou objetivos de projeto (MANDERS-HUITS; ZIMMER 2009); de discordância persistente entre designers e usuários quanto a quais valores são mais importantes (MANDERS-HUITS 2011)(STEEN; VAN DE POEL 2012); ou ainda quando os designers não conseguem implementar todos os valores que descobriram ou a que se comprometeram (FLANAGAN; HOWE; NISSENBAUM 2008).

Em situações de design complexas não há saída óbvia para balancear valores em conflito (MANDERS-HUITS 2011). Contudo, conflitos de valores podem ser enfrentados por meio de um processo de deliberação, o qual permite a retomada da ação (KLAMER 2003). Assim, a proposição e mediação do diálogo entre stakeholders pode ser aplicada como forma de identificar espaços para soluções viáveis (FRIEDMAN; KAHN; BORNING 2006), criando oportunidades para que os 
stakeholders reimaginarem e reengajem-se com seus valores (IVERSEN; HALSKOV; LEONG 2012). Outra estratégia é tornar as diferenças de percepção sobre dados valores explícitas (STEEN; VAN DE POEL 2012).

Os designers têm o desafio de equilibrar seus próprios valores, os dos usuários e outros stakeholders, assim como os da cultura que o cerca (FLANAGAN; HOWE; NISSENBAUM 2005). Dessa forma, é primordial que os mesmos apliquem um processo que seja propício ao debate e integração de valores.

\subsection{Valores vêm do processo}

Segundo Collier e Lakoff (2005 apud SHILTON 2013) uma ideia de prática ou processo compreende um dos componentes principais de um sistema ético. De maneira geral, explorar valores envolve um processo de diálogo e deliberação, no qual trocas verbais, persuasão, conversação e leitura consistem em ferramentas importantes. Deliberações são essenciais ao auxiliar os indivíduos a desenvolver, mudar e, em última instância, determinar seus valores. 0 processo se dá em 3 etapas (valoração, avaliação, e valorização), conforme descrito no Quadro 2. Uma abordagem de design esclarecida quanto a valores deve dar apoio à esses 3 processos, de modo a permitir com que possam ser determinados os agentes, averiguados os valores que os mesmos introduzem, investigar os valores dominantes e estudar como as deliberações influenciam o processo de valoração no ambiente de projeto (KLAMER 2003).

No design, valores não são somente descobertos e clarificados, mas sim consciente e deliberadamente incorporados ao projeto através do método criativo (MANDERS-HUITS; ZIMMER 2009). O valor desejado é usualmente o único fator único fator conhecido no projeto de design, servindo como um guia para os desenvolvimentos subsequentes (DORST 2015). Além disso, valores também são continuamente (e iterativamente) refinados, re-contextualizados e renegociados ao longo do projeto (IVERSEN; HALSKOV; LEONG 2012). Fontes de valores ao longo do processo compreendem a natureza do ambiente de projeto (caracterizada por qualidades como acadêmica, comercial, ativista, etc), assim como itens como os objetivos e hipóteses do projeto (FLANAGAN; HOWE; NISSENBAUM 2008).

Quadro 2 - Etapas do processo de deliberação de valores

\begin{tabular}{|c|c|c|}
\hline Processo & Propósito & Processo \\
\hline Valoração (apreciação) & Atribuir valor & Espontâneo \\
\hline Avaliação & Fazer sentido de uma valoração & $\begin{array}{c}\text { Reflexão consciente sobre } \\
\text { as razões para a } \\
\text { valoração }\end{array}$ \\
\hline Valorização & $\begin{array}{c}\text { Ajustar valores: criação, } \\
\text { aprimoramento e afirmação de } \\
\text { certos valores em detrimento de } \\
\text { outros. }\end{array}$ & Deliberação \\
\hline
\end{tabular}

Fonte: adaptado de Klamer (2003)

Ainda que o design seja uma atividade dirigida por valores, estes comumente permanecem implícitos e inarticulados (STEEN; VAN DE POEL 2012), corroborado pelo fato de metodologias de 
design padrão serem usualmente pensadas para satisfazer requisitos funcionais, sendo estranhas ao mundo dos valores (FLANAGAN; HOWE; NISSENBAUM 2005).

Segundo Van de Poel (2014), o papel desempenhado pelos valores no processo de design é amplamente diverso ao longo das suas diversas etapas e, a partir da identificação das etapas comuns a diversos métodos de projeto (análise, síntese, avaliação e seleção), o autor elenca os diversos papéis desempenhados pelos valores em cada uma delas (Quadro 3). Uma abordagem bem sucedida para integração de valores no projeto de design deve portanto ser abrangente e dinâmica, atentando para uma fluidez característica do trabalho com valores (MANDERS-HUITS; ZIMMER 2009).

Quadro 3 - Papéis desempenhados pelos valores ao longo das etapas do processo de design

\begin{tabular}{|c|c|c|}
\hline Análise & Síntese & Avaliação \\
\hline $\begin{array}{l}\text { Os valores relevantes são } \\
\text { listados e influenciam como o } \\
\text { problema é concebido e } \\
\text { enquadrado. Também são } \\
\text { fontes de requisitos de projeto } \\
\text { e/ou de produto. }\end{array}$ & $\begin{array}{l}\text { Os valores identificados na fase de } \\
\text { análise, agora traduzidos como } \\
\text { requisitos, são incorporados criativa } \\
\text { e diversamente nas soluções } \\
\text { geradas, sempre atentando-se à } \\
\text { potenciais conflitos dos mesmos }\end{array}$ & $\begin{array}{l}\text { Valores são critérios de decisão (sendo } \\
\text { que valores finais devem ser antes } \\
\text { operacionalizados para funcionar como } \\
\text { tal). Usualmente conflitam entre si e/ou } \\
\text { entre objetivos ou requisitos de projeto } \\
\text { ou produto. }\end{array}$ \\
\hline
\end{tabular}

Fonte: adaptado de Van De Poel (2014)

A bibliografia referente a métodos de integração de valores ao projeto de design ou de engenharia estende-se por diversas disciplinas, como antropologia, sociologia, economia e filosofia (KLAMER 2003). Apesar de sua diversidade, todas as abordagens têm em comum a diferenciação de métodos tradicionais de projeto por entenderem os valores como elementos importantes ao longo da integridade da empreitada (MANDERS-HUITS; ZIMMER 2009) e por buscarem integrar valores de uma maneira sistemática, ampla e continuada, no projeto de design (VAN DE POEL 2014)(FRIEDMAN; KAHN 2002)(CUMMINGS 2006). Além disso, algumas abordagens aplicam uma lista específica de valores genéricos, taxonomias ou objetivos de alto nível (HALLORAN et. al 2009).

Dentre as diferenças entre as abordagens, pode ser citado que, enquanto algumas focam em valores pré-selecionados pelos designers, outros elicitam valores para informar o projeto ou destacam a importância de discutir valores com os usuários para incentivá-los a pensar em soluções inovadoras (HALLORAN et. al 2009). Também, algumas abordagens destacam-se para aplicações também específicas, variadas dentro do campo maior do design, como design de software, design arquitetônico, design de políticas públicas, design institucional, dentre outras (VAN DE POEL 2014).

Dentre as diversas abordagens, destacam-se: Design para X (DfX) (VAN DE POEL 2014); Value-Sensitive Design (MANDERS-HUITS 2011); Design para Valores (FLANAGAN; HOWE; NISSENBAUM 2008); Design Participativo (FLANAGAN; HOWE; NISSENBAUM 2008); Design Reflexivo (SENGERS et al. 2005); Design Crítico (SENGERS et al. 2005); Enquadramento (Frame Creation) (DORST 2015); Design Centrado em Valor (Worth-Centred Design) (COCKTON 2008); Avaliação de Tecnologia (Technology Assessment) (VAN DE POEL 2014); Critical Technical Practice (SENGERS et al. 2005); Trabalho Cooperativo Apoiado por Computador (Computer Supported 
Cooperative Work (CSCW)) (SENGERS et al. 2005); Gifted Design (COCKTON 2004); Social Informatics (FRIEDMAN; KAHN; BORNING 2006); Computer Ethics (BREY 2001 apud MANDERS-HUITS 2011); Design Lúdico (SENGERS et al. 2005); Design de Jogos (Game Design) (FLANAGAN; HOWE; NISSENBAUM 2008); e Design Generativo (HALLORAN et. al 2009). Além dessas, existem diversas ferramentas que podem ser aplicadas isoladamente, destacando-se: taxonomias de valores (VOIDA; MYNATT 2005 apud HALLORAN et al 2009); Sondas de Valores (Value probes) (HAINES et al 2007 apud HALLORAN et al 2009); técnica de entrevista laddering (STEEN; VAN DE POEL 2012); Personas (FRIEDMAN; KAHN; BORNING 2006) e Alavancas de Valores (SHILTON 2013).

\section{Resultados e Discussão}

Valores só surgem a partir da experiência (KLAMER 2003). Dessa forma, estão por todo lugar, mas, ao mesmo tempo, não estão em lugar algum. Através dessa "onipresença implícita", valores estão incorporados implicitamente nos artefatos e são trazidos à tona somente por meio do engajamento na criação e uso desses mesmos produtos, sistemas e/ou experiências.

Nesse contexto, na medida em que áreas como a sociologia proporcionam entendimentos para formatar as inovações aos contextos de uso e outras, como a psicologia, apóiam o refinamento e avaliação das soluções, o designer pode e deve atuar na mediação da relação dos usuários e outros stakeholders com os objetos, apoiando-os e engajando-os na entrega de um valor proposital desejado (COCKTON 2004)(IVERSEN; HALSKOV; LEONG 2012). Para tal, no entanto, é necessário entender quais as fontes dos valores morais no design, na medida em que, a partir do conhecimento desses parâmetros, é possível modificá-los de modo a otimizar o processo.

Como resultado específico desta pesquisa, identificaram-se e exploraram-se 4 grandes grupos de fontes de valores no processo de design: o artefato; o designer; o usuário e outros stakeholders; e o processo. É válido destavar que todos os grupos de fontes de valores, apesar de distintos, apresentam interseç̧ões. Similarmente ao próprio processo colaborativo, em que o significado é cocriado entre os participantes, os valores advindos do artefato, do designer, dos usuários e outros stakeholders e do processo influenciam-se mutuamente.

De maneira a exemplificar essa relação, a figura 1 apresenta os grupos de fontes de valores identificados, destacando, de maneira visual, o papel intermediador dos artefatos entre designer e usuário, assim como a imersão dessas três fontes de valores no processo de design colaborativo. De maneira geral, é possível inferir que, na medida em que o designer incute seus valores pessoais e profissionais no processo que formata e nos artefatos que projeta, dos quais lança mão para intermediação do processo colaborativo junto dos usuários e outros stakeholders, estes últimos recebem os valores incutidos e os reinterpretam. Assim, devolvem-los, reapreciados, em um processo dialógico de consolidação gradual de valores prioritários ao contexto específico do projeto. 
Figura 1 - Esquematização de fontes de valores no processo de design colaborativo

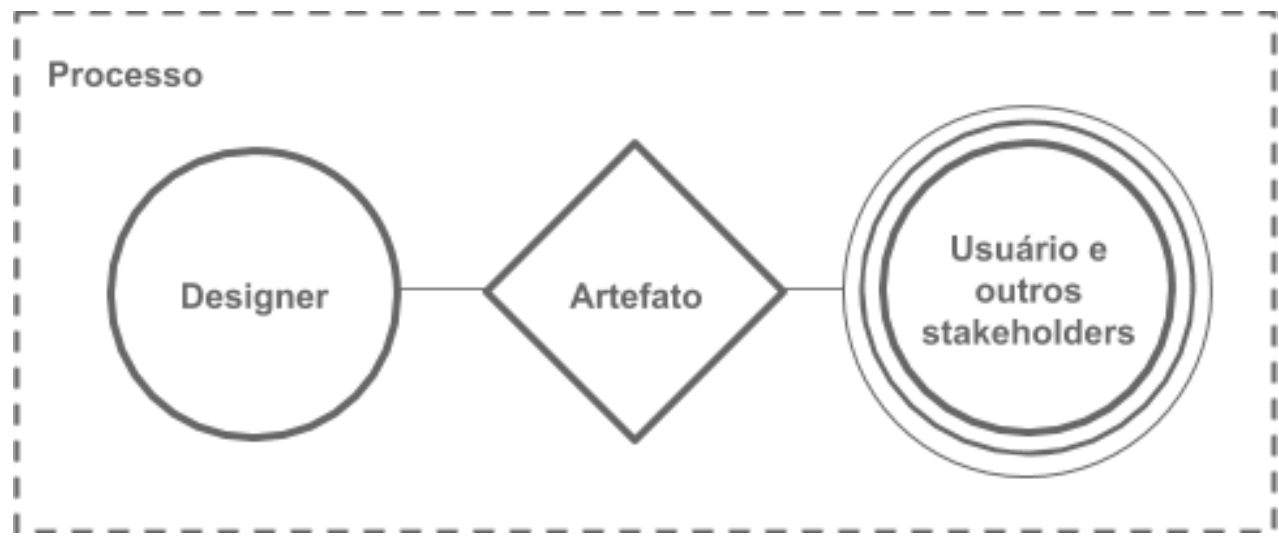

Fonte: o autor

\section{Considerações Finais}

A presente pesquisa buscou, através de uma pesquisa bibliográfica, estabelecer um panorama quanto às principais fontes de valores morais no Design Colaborativo. No entanto, denota-se que o estudo carece de uma experimentação prática. Estudos de caso embasados no modelo de fontes de valores aqui apresentado poderiam auxiliar a validação e consolidação dos resultados encontrados.

\section{Referências}

BROBERG, O.; ANDERSEN, V.;SEIM, R. Participatory ergonomics in design processes: The role of boundary objects. Applied Ergonomics, 42(3), 464-472, 2011.

COCKTON, G. Value-centred $\mathrm{HCl}$. Proceedings of the Third Nordic Conference on Human-Computer Interaction - NordiCHI '04, 149-160, 2004.

COCKTON, G. Designing Worth - Connecting Preferred Means to Desired Ends. Interactions, 15(4), 54-57, 2008.

CUMMINGS, M. L. Integrating ethics in design through the value-sensitive design approach. Science and Engineering Ethics, 12(4), 701-715, 2006.

DITTRICH, Y.; ERIKSÉN, S.; WESSELS, B. Why the specific is crucial for participatory design research. Scandinavian Journal of Information Systems, 26(1), 29-56, 2004.

DORST, K. Frame Creation and Design in the Expanded Field, (1), 22-33, 2015.

FLANAGAN, M.; HOWE, D. C.; NISSENBAUM, H. Values at Play: Design Tradeoffs in Socially-Oriented Game Design. Proceedings of the SIGCHI Conference on Human Factors in Computing Systems. ACM, 751-760, 2008.

FRIEDMAN, B.; KAHN, P. Value sensitive design: Theory and methods. University of Washington Technical, (December), 1-8, 2002.

FRIEDMAN, B.; KAHN Jr., P. H.; BORNING, A. Value Sensitive Design and Information Systems. Human-Computer Interaction and Management Information Systems: Foundations, 1-27, 2006. 
HALLORAN, J. et al. The value of values: Resourcing co-design of ubiquitous computing. CoDesign, 5(4), 245-273, 2009.

IVERSEN, O. S.; HALSKOV, K.; LEONG, T. W. Values-led participatory design. CoDesign, 8(2-3), 87-103, 2012.

KLAMER, A. A pragmatic view on values in economics. Journal of Economic Methodology, 10(2), 191-212+271, 2003.

KNOBEL, C.; BOWKER, G. C. Values in design. Communications of the ACM, 54(7), 26, 2011.

MANDERS-HUITS, N.; ZIMMER, M. Values and Pragmatic Action: The Challenges of Introducing Ethical Intelligence in Technical Design Communities. International Review of Information Ethics, 10, 37-44, 2009.

MANDERS-HUITS, N. What Values in Design? The Challenge of Incorporating Moral Values into Design. Science and Engineering Ethics, 17(2), 271-287, 2011.

OREG, S.; NOV, O. Exploring motivations for contributing to open source initiatives: The roles of contribution context and personal values. Computers in Human Behavior, 24(5), 2055-2073, 2008.

SIEKMANN, J. Lecture Notes in Artificial Intelligence. Lecture Notes in Computer Science (Vol. 1529), 1998. (http://portal.acm.org/citation.cfm?id=945844)

SHILTON, K. Values Levers: Building Ethics into Design. Science Technology and Human Values, 38(3), 374-397, 2013.

SENGERS, P. et al. Reflective design. Proceedings of the 4th Decennial Conference on Critical Computing between Sense and Sensibility - CC '05, 49, 2005.

STEEN, M.; VAN DE POEL, I. Making values explicit during the design process. IEEE Technology and Society Magazine, 31(4), 63-72, 2012.

SPENCER-OATEY, H. What is Culture? A Compilation of Quotations. GlobalPAD Core Concepts, 2012.

VERBEEK, P.-P. Materializing Morality. Science, Technology, \& Human Values, 31(3), 361-380, 2006.

VAN DE POEL, I. Design for Values in Engineering. Handbook of Ethics, Values, and Technological Design, 1-20, 2014. 